\title{
La revue « radioprotection », 1966 à 1999
}

\author{
D. BLANC ${ }^{1}$
}

(Manuscrit reçu le 18 novembre 2004, accepté le 21 mars 2004)

RÉSUMÉ Dans le $n^{\circ} 1$ du volume 26 de « radioprotection » (1991), j’évoquais la naissance de la revue, en novembre 1966, et je rappelais les sommaires des cinq premiers numéros. La création d'une rubrique d'inspiration historique me permet de présenter, de manière détaillée, trente trois ans d'existence de la revue. L'exposé est divisé en cinq tranches chronologiquement successives, chacune correspondant à un responsable : 1966-1979 (13 ans), Henri François, Norbert Chassende Baroz, Anne-Marie Beau ; 1979-1982 (3 ans), André Teste du Bailler et Anne-Marie Beau ; 1982-1987 (5 ans), Pierre Bovard et Anne-Marie Beau ; 1987-1996 (9 ans), Daniel Blanc et Anne-Marie Beau (jusqu'à la fin de 1995) ; depuis 1996, Henri Métivier et un comité de rédaction secondé par une équipe.

ABSTRACT “Radioprotection" journal, 1966 to 1999.

In the number 1 of volume 26 of Radioprotection (1991), I evoked the birth of the review, in November 1966, and I pointed out the contents of the five first numbers. The creation of a historical heading of inspiration enables me to present, in a detailed way, thirty-three years of existence of the review. The talk is divided into five parts chronologically presented, each one corresponds with a person in charge: 1966-1979 (13 years), Henri François, Norbert Chassende-baroz, Anne-Marie Beau; 1979-1982 (3 years), André Teste du Bailler and Anne-Marie Beau, 1882-1987 (5 years), Pierre Bovard and Anne-Marie Beau, 1987-1996 (9 years), Daniel Blanc and Anne-Marie Beau (until the end of 1995); since 1996, Henri Métivier assisted by an editorial board.

Key words: SFRP / journal / history

\section{Avant propos}

On imagine mal une société scientifique comme la SFRP sans une revue, qui constitue le témoignage matériel de ses travaux et de son activité. En effet, dès la fin de 1965, se fait sentir le besoin que la nouvelle société se manifeste à l'extérieur, ne serait-ce qu'à cause de son appartenance à l'IRPA. On met en place un bulletin, et le terme de radioprotection s'impose tout naturellement. On crée donc le bulletin de la Société française de radioprotection.

\section{Radioprotection, bulletin de la société française de radioprotection}

On prévoyait de faire paraître le premier numéro de la revue dès la rentrée des congés de l'été 1966 ; des retards dans la fabrication firent que ce numéro fut

\footnotetext{
1 Au nom de la SFRP.
} 
distribué au début de novembre. Il s'ouvre sur l'éditorial du professeur Louis Bugnard, qui écrit : "[...] Il fallait à cette jeune société un moyen de liaison qui lui permette d'éclairer les esprits par la diffusion des connaissances et qui constitue pour ses membres un point de rencontre leur donnant la possibilité d'exposer et de confronter les résultats les plus récents obtenus dans les domaines si divers de la radioprotection. C'est désormais chose faite [...]».

Puis un communiqué signé collégialement par le conseil d'administration présente la SFRP. On y trouve la définition du domaine : «[...] La radioprotection apparaît comme une science multidisciplinaire : physique, électronique, radiochimie, métrologie, pédologie, hydrogéologie, sédimentologie, océanographie, agronomie, écologie, biologie et sciences médicales, sont, à des titres variés, concernées par les multiples aspects scientifiques et techniques de la protection de l'homme et du milieu vis à vis des rayonnements ionisants [...] ».

Le caractère multidisciplinaire de la revue se manifeste dès les six articles de ce numéro; il se confirmera, et cela implique qu'il sera toujours délicat de lui donner un bon équilibre rédactionnel, et de le garder.

La mention «bulletin de la SFRP » disparaît en 1971, probablement pour des raisons liées à la TVA [1]*.

\section{Un coup d'œil rapide}

La revue repose sur trois bases.

Le responsable scientifique de la revue, président de la commission des publications, puis de la commission de la revue, enfin du comité de rédaction. Nommé par le président de la SFRP, il est responsable devant le conseil, qui peut éventuellement mettre fin à ses fonctions. Invité à participer aux délibérations du conseil, il peut être amené à répondre à des questions ou à des critiques. Il présente devant l'assemblée générale un rapport annuel.

Un opérationnel travaille en liaison étroite avec le responsable scientifique. Il est chargé de la réalisation éditoriale de la revue ; il doit veiller au «timing » des opérations et assurer une liaison constante avec la société qui édite la revue. Nous avons eu la chance que ce travail soit effectué par deux personnes de très haute qualité, le général N. Chassende Baroz (1966 à 1974) et Madame A.-M. Beau (1974 à 1995).

\footnotetext{
* Voir note importante en fin d'article
} 
Le secrétaire général de la SFRP assure la liaison permanente avec le conseil et le président de la SFRP. Il assiste aux réunions de la rédaction ; avec le trésorier, il contrôle la bonne marche du système au plan financier. Il a pris la responsabilité de la partie «RP magazine» de la revue, crée pour donner des informations d'ordre général, telles que nouvelles de la société, de la radioprotection dans le monde, des annonces et des comptes rendus de congrès, l'évolution de la réglementation, etc.

Au-delà de cette organisation se trouve l'éditeur, qui a en charge la fabrication et la diffusion de chaque numéro. Il est directeur de la publication ; autrement dit, il est propriétaire des textes publiés, et seul habilité à négocier des « copyrights ». La jeune SFRP l'ignorait, ce qui lui a valu des désagréments, comme nous le verrons. Nous avons eu affaire à trois éditeurs : novembre 1966 à décembre 1981, (15 ans), Dunod, puis Bordas-Dunod ; janvier 1982 à décembre 1991, (10 ans), le GEDIM, à Saint-Étienne ; depuis janvier 1992, EDP Sciences (anciennement Éditions de Physique), aux Ulis.

Les rapports avec l'éditeur ont connu des hauts et des bas. Très variables et parfois mauvais avec Dunod puis Bordas-Dunod, ils furent excellents avec le GEDIM, et ont pris une bonne vitesse de croisière avec EDP Sciences. Enfin, l'éditeur est chargé de recueillir les abonnements extérieurs aux membres de la SFRP.

\section{De 1966 à septembre 1979 : Henri François avec Norbert Chassende Baroz, puis Anne-Marie Beau}

\subsection{Mise en place de la commission des publications}

En même temps qu'il crée la revue, le conseil de la SFRP met en place une commission des publications, chargée de l'ensemble des écrits de la SFRP (actes des congrès, des journées, brochures, mais surtout la revue). Voici la liste de ses membres (ceux qui sont membres du conseil ont leurs noms soulignés) : H. François,

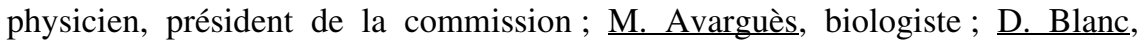
physicien; P. Bonet-Maury, pharmacien; $\underline{\mathrm{N}}$. Chassende Baroz, pharmacien;

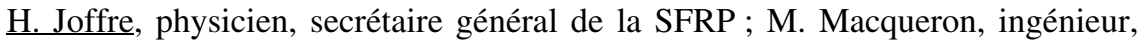
ancien officier de marine ; J. Manquené, médecin.

H. François est membre du conseil de 1968 à 1971, J. Manquené de 1967 à 1970. À l'origine, sur huit des membres, cinq font partie du conseil, sept, deux ans plus tard. Cet aspect «politique» s'effacera ensuite, progressivement. Après la disparition de la commission en 1993, aucun des membres du comité de rédaction 
n'est membre du conseil. Le secrétaire général continuera à participer, es-qualité, aux travaux du comité de rédaction. En face de la commission, se trouve Georges Dunod, président des éditions Dunod, en tant que directeur de la publication.

H. François, d'origine universitaire, est ingénieur au CEA. Il dirige une section de dosimétrie, basée au centre d'études de Fontenay-aux-Roses. C'est avant tout un chercheur, spécialiste de la thermoluminescence. N. Chassende Baroz préside à la réalisation matérielle de la revue ; il excelle dans ce rôle, où il est secondé par M. Macqueron, ingénieur au service technique d'études de protection du CEA.

Dès ses débuts, la revue est trimestrielle ; sa parution est prévue en mars, juin, septembre et décembre. Durant cette première période, les dates de parution furent peu respectées, mais sa périodicité n'a jamais changé, ni son format.

\subsection{Le partage des tâches}

Un comité de rédaction est créé en 1968. Le président Maurice Delpla l'explique lors de l'assemblée générale (AG) du 15 mai 1968, (Delpla, 1968) : « [...] La commission des publications a tenu autant, sinon d'avantage, de réunions que le conseil d'administration auquel beaucoup de ses membres appartiennent. Pour faire front, pour supporter l'accroissement de la charge qui résulte de la prise à notre compte de la publicité dans la revue, Mr Chassende Baroz vient de substituer au « secrétariat de publication du bulletin » un «comité de rédaction » beaucoup plus étoffé que le secrétariat de publication, mais, comme lui, étroitement associé à la commission des publications [...]».

Dirigé par N. Chassende Baroz, le comité de rédaction se divise en deux sections. L'une, «publications-informations », est dirigée par Mr R. Hantz, l'un des collaborateurs du Dr J. Manquené (service médical du centre de Fontenay-auxRoses), avec M. Macqueron, qui quitte alors la commission des publications. L'autre, « publicité », est confiée à M. Gras (SCRGR), entouré de D. Blanc, J.-M. Chinardet, M. Diéval, H. François, R. Hantz et H. Joffre. Son but est de trouver des annonceurs pour la revue.

N. Chassende Baroz ne souhaite pas coiffer les deux sections. En 1969, l'organisation devient : comité de rédaction (MM. Chassende Baroz, Hantz et Macqueron) et comité de publicité, dirigé par H. Joffre, chef du SPR de Saclay, avec MM. Blanc, Chinardet, Diéval, François, Gras et Hantz.

La commission des publications grossit progressivement, et $\mathrm{H}$. François constitue un comité scientifique de la revue, plus restreint ( 7 ou 8 membres).

Les rapports avec le conseil d'administration sont détendus. Une modification — mineure - est présentée par Francis Duhamel, président sortant, à l'AG du 
5 juin 1970 (Duhamel, 1970) : «les présidents des commissions sont désignés chaque année dans le courant du semestre qui suit l'assemblée générale. Nous avons introduit un additif à cette réglementation : la désignation ainsi faite par le conseil d'administration prendra effet à compter de l'exercice suivant. De cette manière, le titulaire, d'une part disposera d'au moins six mois pour préparer son entrée en fonction, d'autre part commencera à exercer ses fonctions sous la présidence suivant celle où sa désignation aura eu lieu. Nous pensons faciliter ainsi la coopération entre les différentes personnalités qui dirigent les organes de notre société ».

Compliqué ? Certes, mais le passage ultérieur à deux ans du mandat du président de la SFRP va rendre sans effet cette mesure, les responsables des susdits « organes » étant remis en question lors de chaque nouvelle présidence. Résumons les choses pour les lecteurs étourdis : travaillent en parallèle, dans le cadre d'une commission des publications très élargie, voire diffuse, le comité scientifique de la revue (H. François), le comité de rédaction (N. Chassende Baroz), le comité de la publicité (H. Joffre), qui devient en 1971 « relations avec l'industrie », et disparaît à la fin de cette même année. Pour coordonner tout cela et assurer la liaison avec le conseil d'administration, le président nomme un rapporteur général (joli terme, n'est il pas vrai). Ce rapporteur deviendra président de la commission des publications, et cela va durer jusqu'à la fin de 1973. Ce rapporteur, c'était moi, et je voudrais préciser les choses : j'étais flatté de devenir général, mais cela n'allait pas au-delà ; le système fonctionnait bien, et il n'était absolument pas question d'intervenir de manière " hiérarchique » au milieu de bénévoles. J'ai décidé de ne rien faire. Contrairement à ce qu'indiquent alors les textes, la direction effective est demeurée entre les mains de H. François et de N. Chassende Baroz. Mon rôle de rapporteur, puis de président, était purement « convivial ». Plus importante est la nomination par le conseil, le 13 janvier 1971, de P. Ballereau «délégué du secrétaire général pour la documentation ». Ce titre pompeux (la SFRP adorait cela, à l'époque) cache un travail efficace. Pierre Ballereau continue à être rapporteur des séances du conseil, et de celles du club « histoire ».

\subsection{Les problèmes posés par l'éditeur}

Notre éditeur durant cette période, et une grande partie de la seconde (jusqu'à décembre 1981), est Dunod, nom prestigieux dans l'édition scientifique et technique. À partir de 1970, se produisent des «concentrations » successives : Dunod avait absorbé Gauthier-Villars, mais il est à son tour absorbé par Bordas à la fin de 1971. Ultérieurement, Bordas entrera dans un groupe plus important. Vu ses difficultés, l'éditeur met sa marge bénéficiaire au premier plan de ses préoccupations; il cherche constamment à augmenter le prix de vente de la revue. Le 2 avril 1971, N. Chassende Baroz annonce au conseil que Dunod impose à la 
SFRP une facturation de chaque exemplaire à $38 \mathrm{~F}$, à compter du $1^{\text {er }}$ juillet 1971 pour les abonnés, et du $1^{\text {er }}$ janvier 1972 pour les sociétaires [1]. C'est une augmentation de $15 \%$. Après études et consultations, le conseil se résigne à accepter cette augmentation, faute de pouvoir trouver mieux. Cela conduit à envisager une cotisation de 60 francs, revue comprise, en 1972 [2].

En octobre 1971, le conseil apprend que, malgré la bonne volonté de la SFRP, Dunod considère l'édition de la revue comme non rentable. Le rachat de Dunod par Bordas, à la fin de 1971, complique les choses : Dunod (qui n'a gardé que son nom) accepte de continuer, à condition que seize pages d'articles soient supprimées dans chaque numéro, mais avec possibilité de rachat. Par ailleurs, l'éditeur se chargerait lui même de la publicité, avec la collaboration d'agences spécialisées ; une ristourne, accordée à la SFRP, lui permettrait de récupérer, ultérieurement, les pages perdues [3].

Un projet de convention, daté du 5 avril 1972, est présenté à la commission des publications : l'éditeur prévoit une revue de 256 feuillets annuels (recto et verso), en quatre numéros de 64 feuillets; la publicité est confiée à Dunod. À partir de la $28^{\mathrm{e}}$ page de la revue, chaque page de publicité donne droit à 6 pages de texte ; l'injection par la SFRP de 4180 francs par an lui permettrait de racheter, au titre de compte d'auteur, les pages perdues.

Le conseil ne saisit pas que l'association se trouve spoliée en ce qui concerne la publicité, qui est, ou peut être, une source importante de financement. En fait, c'est une dernière clause qui soulève sa — légitime — colère : Dunod s'attribue la propriété du titre « radioprotection » et demanderait une indemnité de 9000 francs pour le rachat du titre, en cas de changement d'éditeur [4]. On reprend les discussions ; entre propositions et contre-propositions, plus d'une année s'écoule, les accords anciens étant prorogés durant cette période. Finalement, on signe, le 20 novembre 1973 [5], un accord relativement satisfaisant, maintenant la propriété de la SFRP sur le titre « radioprotection».

Toutes ces difficultés ont pour conséquence un retard progressivement croissant de la sortie des numéros de la revue ; ainsi, le n ${ }^{\circ} 4$ de 1973 paraît en mai 1974.

\subsection{Des difficultés de parcours...}

Dans son rapport moral devant l'AG du 13 juin 1974, le président Albert Gey résume clairement les choses et apporte des précisions importantes (Gey, 1974): " [...] La revue radioprotection est l'affaire de la commission des publications, et notamment de son président, Henri François. Vous vous êtes peut être aperçus des difficultés qui ont quelque peu perturbé la régularité de sa 
LA REVUE « RADIOPROTECTION », 1966 À 1999

publication. Je m'empresse de le dire, ces difficultés sont, pour le passé, aplanies. Et grâce, notamment, à l'activité inlassable comme au dévouement désintéressé à cette cause de MM. Chassende Baroz, Hantz, Bittel et Joffre, les retards ont pu être comblés. La cause majeure de ces retards, il faut l'avouer, c'est la négociation laborieuse d'un protocole avec l'éditeur, protocole rendu nécessaire par des changements de structures externes à la Société. C'est pourquoi, j'ai, dès ma prise de fonctions, assigné à la signature de ce protocole, la priorité des priorités. Grâce au dynamisme constant et organisé d'un membre du conseil d'administration, Mr Portal, ce protocole a été signé par les deux parties en novembre 1973. Plus qu'un accord, il faut le considérer comme une base susceptible d'être améliorée. Il ne faut pas se leurrer, nous aurons dans l'avenir des difficultés à surmonter et, par dessus tout, le prix de l'abonnement et la qualité de la revue. Ce protocole aura au moins le mérite de couvrir sensiblement tous les aspects, les avantages comme les contraintes, d'une collaboration avec n'importe quel éditeur. Il permet donc des choix objectifs entre des prestations différentes, et, si on le voulait, on pourrait s'en servir comme base de discussion et de comparaison entre des offres différentes. Il montre aussi, à l'évidence, que l'édition d'une revue fait intervenir une nuée de paramètres, parmi lesquels les paramètres administratifs ou contentieux sont loin d'être négligeables. Je souhaite que les liaisons nécessaires qui se sont établies à cette occasion entre le conseil d'administration et sa commission des publications se perpétuent dans l'avenir et se renforcent, si faire se peut.

Second écueil rencontré par la commission des publications : le portefeuille d'articles à publier. Deux doléances se sont principalement manifestées, l'une concernant leur quantité, l'autre concernant leur variété. Concernant le nombre d'articles, la commission se plaint d'avoir un portefeuille en forme d'accordéon, tantôt trop plein, tantôt pas assez. Quand il est trop plein, les articles les plus récents vieillissent faute de place immédiatement disponible dans l'édition. Quand il est trop vide, il faut « sonner » à toutes les portes pour le regarnir. Concernant leur variété, certains sociétaires regrettent de ne pas trouver dans la revue ce qu'ils souhaiteraient : par exemple des articles sur la sûreté des installations, sur la surveillance opérationnelle, sur les incidents et leurs enseignements, etc. La revue étant faite pour, mais aussi par les sociétaires, je souhaite que chacun se sente concerné et, qu'avant de recevoir, il songe à donner. Je souhaite aussi qu'au sein même de la commission, des membres se fassent prospecteurs permanents d'articles par discipline, et à raison d'un à deux coups de fil par mois; cela allègerait d'autant la tâche du président de la commission. Je souhaite enfin que personne ne s'étonne des délais - parfois longs - entre le bon à tirer et la parution, car la rédaction et l'édition sont œuvres de longue haleine, et la qualité comme la tenue de la revue sont une caution de survie de la Société». 
Au milieu de l'année 1974, survient un événement qui va s'avérer déterminant pour la bonne marche de la revue : A.-M. Beau, responsable de la documentation au département de protection radiologique du CEA, à Fontenay-aux-Roses, accepte de devenir secrétaire de la commission des publications, et secrétaire de la rédaction. Agissant immédiatement, elle enlève à la commission pas mal de soucis. Elle devait garder ces fonctions durant plus de vingt ans. La régularité de la parution de la revue, est due pour beaucoup à son efficacité et à son sens des réalités.

Les difficultés que rencontre la revue ne sont pas toutes aplanies, il s'en faut. Dans sa lettre de vœux pour 1976, le président Henry Letard écrit : «Notre revue Radioprotection reste toujours pour nous un souci. Il devient en effet urgent de pouvoir en étendre la diffusion. Pour parvenir à ce résultat, il serait nécessaire que la commission des publications puisse disposer d'un certain nombre d'articles qui, tout en conservant un niveau scientifique de valeur, aborderaient des sujets plus larges, et moins hautement spécialisés. Votre président ne peut que vous exprimer le vœu qui en a été formulé par le conseil d'administration de la Société, en vous demandant une aide personnelle directe par l'envoi d'un ou de plusieurs articles rédigés dans le sens indiqué plus haut. Le maintien de la publication de notre revue peut être à ce prix, et je souhaite que nous trouvions auprès de beaucoup d'entre vous l'aide qui nous est indispensable dans ce domaine ».

Son rapport devant l'AG du 2 juin 1976 est moins pessimiste (Letard, 1976) : « [...] J'en viens maintenant à une forme importante de l'activité de notre Société, la revue Radioprotection. Au cours des rapports moraux que nous avons entendus ces deux années précédentes, il nous a été signalé les difficultés auxquelles devait faire face la commission des publications que dirige Mr François. La signature d'un protocole avec l'éditeur avait été la première étape nécessaire à l'aplanissement des difficultés; néanmoins, les retards acquis ne pouvaient être comblés de façon immédiate. Aujourd'hui, je puis vous annoncer que, non seulement tous les retards sont rattrapés, mais que la situation de la revue au plan de la rédaction est bonne, puisque le premier numéro 1976 était sorti pour le congrès SFRP du mois de mars, le numéro 2 dont le bon à tirer est depuis plusieurs jours entre les mains de l'éditeur, sortira pour le 15 juin. Les épreuves du numéro 3 ont été remises à l'éditeur le 8 mai, et la sortie de ce numéro est prévue pour le 14 septembre. Je crois, Messieurs, que nous pouvons apprécier à sa juste valeur l'effort fourni par la commission des publications. Vous me permettrez d'associer particulièrement à cet effort Madame Beau dont l'efficace dévouement a certainement constitué une contribution importante. Je serais incomplet si, dans le cadre de notre revue, je ne signalais pas la demande de subvention de 20000 francs formulée en juin 1975 par Mr Stolz auprès du CNRS. Cette demande 
a reçu une réponse partiellement positive, puisqu'une subvention de 5000 francs nous a été effectivement accordée. Nous espérons que, formulant maintenant une nouvelle demande, l'allocation qui pourra nous être versée sera telle qu'elle nous permettra un effort promotionnel important.

Par ailleurs, la question pourrait se poser d'une ouverture de la revue et de la société sur des risques moins spécifiquement liés aux rayonnements ionisants ».

La situation de la revue se stabilise, en ce qui concerne la régularité de publication, mais aussi le nombre des manuscrits : « [...] Il m'apparaît important de vous informer que notre portefeuille d'articles est bien pourvu et que, depuis le $n^{\circ} 2$ (de 1977), des articles sont publiés sur des sujets relatifs aux rayonnements non ionisants. Par contre, nous manquons toujours d'articles techniques sur la radioprotection (opérations de surveillance et d'intervention, résultats de contrôle d'installations nucléaires, etc.) ", écrit le président Lucien Fitoussi (Fitoussi, 1977), dans son rapport moral devant l'AG du 21 juin 1977.

\section{5. ...et la durée des fonctions du responsable de la revue}

Le calme n'est qu'apparent. En fait, les présidents, en particulier A. Gey et H. Letard, sont agacés. La raison est la durée des fonctions de H. François, dix ans en 1976. D'un caractère très indépendant, il ne tient guère compte des avis du conseil d'administration et de son président. Cependant, sa politique éditoriale est bonne et volontariste ; on lui doit la publication d'articles de qualité, en anglais, mais cela se fait contre la volonté du conseil, qui, dans sa grande majorité, est hostile à l'introduction de l'anglais, et se déclare pour la défense du français.

Quelques précisions à ce sujet. Voici les noms d'auteurs étrangers durant la présidence de H. François : W.S. Snyder (États-Unis), G. Busuoli (Italie), J.E. Turner (États-Unis), M. Jensen et J.O. Mathiesen, R. Katz (États-Unis), etc. Il y a même un très bon article d'auteurs français, publié en anglais en 1979 (Mme M. Dardalhon et MM. A.-J. Berteaud et D. Averbeck). Mais de telles publications déplaisent au conseil.

Par ailleurs, le nombre strictement contingenté des pages de la revue contribue à en rejeter les notes d'information. Le conseil décide de mettre en place un bulletin interne. La maquette du $\mathrm{n}^{\circ} 1$ du bulletin d'information de la SFRP est distribuée aux membres du conseil lors de sa réunion du 9 février 1978. Le bulletin n'est distribué qu'aux membres de la société. Il est placé sous la responsabilité du secrétaire général, qui en assure la mise en pages et la rédaction, avec l'aide de M. Gras. La périodicité du bulletin est variable, et fonction de l'urgence et du volume des informations. Il est paradoxal que, bien que le budget de la revue soit un souci, on ait créé une seconde source de déficit. 
Ce bulletin marque un besoin, presque une nécessité, mais il est très regrettable qu'il soit dissocié de la revue. Tout s'arrangera quand on changera d'éditeur, et l'on en arrivera à la solution optimale d'un magazine couplé à la revue.

Le 10 novembre 1978, le conseil discute du renouvellement de l'équipe qui anime la revue depuis sa création: "le président pense que l'inamovibilité des commissions présente plus d'inconvénients que d'avantages, compte tenu notamment du déséquilibre dû à la rotation rapide des administrateurs et des membres du bureau. Il paraît donc souhaitable de modifier le règlement intérieur pour institutionnaliser le renouvellement des commissions » [6].

Le conseil prend les deux décisions suivantes :

(a) le conseil d'administration approuve (unanimité moins une voix) le principe d'une modification du règlement intérieur limitant la durée du mandat des présidents des commissions ; le nouveau texte sera mis au point en accord avec la commission juridique et du règlement ;

(b) Henri François est élu, à la majorité, président de la commission de la revue pour l'exercice 1978/1979.

En septembre 1979, H. François renouvelle sa candidature à la présidence de la commission de la revue. A. Teste du Bailler, administrateur, pose aussi sa candidature. La question est inscrite à l'ordre du jour du conseil du 24 septembre 1979 [7]. Une longue discussion aboutit au résultat suivant: 14 votants, A. Teste du Bailler ne participant pas au vote, A. Teste du Bailler, 13 voix ; H. François, 1 voix.

\section{D'octobre 1979 à septembre 1982 : André Teste du Bailler avec Anne-Marie Beau}

Les responsabilités de A. Teste du Bailler se terminent en septembre 1982, car il est alors président élu de la SFRP, et doit se préparer à son futur mandat de président. En 1980, le conseil nomme N. Chassende Baroz et H. François « créateurs de la revue », ce qui leur confère une présence permanente dans la commission de la revue.

\subsection{Une enquête auprès des lecteurs...}

Devant l'assemblée générale (AG) du 27 juin 1979, le président Philippe Beau avait indiqué que : "notre éditeur nous a proposé le lancement d'une enquête sur les besoins et les attentes des milieux professionnels confrontés à des problèmes 
de radioprotection, dont nous allons mettre au point avec lui les modalités dans les mois qui suivent ».

L'une des premières tâches du président de la commission est de mettre en chantier un questionnaire, qui accompagne la livraison des numéros 1 et 2 de 1980 : "la revue répond-elle aux besoins de ses lecteurs? Doit-on la modifier pour améliorer et accroître son audience auprès des scientifiques, des industriels, des médecins, du grand public?».

Pour la première fois depuis la création de la revue, son responsable s'adresse aux lecteurs dans un éditorial ( $\mathrm{n}^{\circ} 1$ de 1980). Cela va devenir une tradition. Pour sa part, A. Teste du Bailler écrira huit éditoriaux. Nous citons son premier texte : ses propos vont tout à fait dans le sens souhaité par le conseil : «[...] Nous voudrions être plus clairs, plus complets, plus actuels, plus utiles aussi à ceux qui conduisent ou exploitent des installations émettant des rayonnements. Pour cela, nous ouvrirons nos colonnes, en particulier dans un courrier des lecteurs, à tous ceux qui souhaitent apporter un point de vue ou une contribution ».

Le questionnaire est simple, y répondre demande peu de temps, et pourtant l'enquête est un échec, car seulement $5 \%$ des lecteurs répondent. Faut-il en déduire que tous les autres sont parfaitement satisfaits?

Dans son éditorial du $\mathrm{n}^{\circ} 4$ de 1980, A. Teste du Bailler résume les enseignements qu'il a tirés du questionnaire : " $[\ldots] \mathbf{1}^{\circ}$ Les lecteurs sont, en général, satisfaits sur la forme de la revue (70\%) mais pas sur le fond $(30 \%) .2^{\circ}$ Ils sont favorables à développer l'information d'un public large $(60 \%)$ et à traiter la sûreté nucléaire $(70 \%) .3^{\circ}$ Ils souhaitent que soient ouvertes de nouvelles rubriques comme le courrier des lecteurs, les lettres à l'éditeur, un éditorial plus régulier. $4^{\circ}$ D’une façon générale, les lecteurs attendent de la revue qu'elle soit un organe d'échange et de communication, qu'elle transmette l'actualité, qu'elle contienne des articles plus clairs, plus concis, traitant des problèmes concrets de la radioprotection ».

\section{2. ... et la suite}

Devant l'AG du 20 juin 1980, le président Raymond Bardoux consacre un long passage à la revue et à ses problèmes. Il résume les exposés faits devant le conseil par le responsable de la revue. En voici un extrait : «[...] $3^{\circ}$ Situation financière. Nous en retiendrons deux aspects contradictoires, une forte augmentation $d u$ prix de l'abonnement (de l'ordre de $300 \%$ entre 1975 et 1980); un bilan déficitaire aux dires de l'éditeur, malgré l'image de marque flatteuse de Dunod, éditeur de nombreuses publications scientifiques ». 
Le conseil a mis en place, en 1980, un groupe de réflexion sur la revue, qui aborde les problèmes relevant du contenu même de la revue. Écoutons à nouveau R. Bardoux : "le caractère des articles ne doit être, ni exclusivement théorique, $n i$ exclusivement technique ou pratique, mais l'ensemble de ces caractères doit être équilibré de façon harmonieuse. On doit pouvoir trouver dans chaque numéro : (1) un ou deux articles scientifiques de haut niveau, faisant le point sur une question et plusieurs articles techniques courts, donnant des indications pratiques (chiffres, courbes); (2) des «lettres à l'éditeur » traitant de problèmes concrets : analyse d'incidents, résultats d'essais, et un courrier des lecteurs, éventuellement ouvert à la contestation antinucléaire, pourvu qu'elle garde un aspect objectif scientifique; (3) des rubriques régulières, informant la communauté des radioprotectionnistes de toutes les nouvelles concernant travaux et groupes de travail, nouvelles réglementations, informations techniques sur les matériaux nouveaux (normalisation, fiches techniques, voire publicité informative ».

Ce n'est pas très éloigné de la structure actuelle de la revue.

Parallèlement, Guy Uzzan, secrétaire général, indique à l'AG que "notre bulletin interne, les nouvelles de la radioprotection, constitue, de par sa nature, le vecteur efficace et particulièrement bien adapté de la diffusion rapide de l'information auprès de nos membres, touchant les divers secteurs de la radioprotection ».

Au total, la revue bute sur deux écueils: (1) le manque de manuscrits de qualité, (2) des problèmes de budget. Ils conduisent à des effets analogues.

\subsection{Où il est question de l'éditeur}

Les rapports avec Dunod sont médiocres, et tendent à s'envenimer. Nous avons devant nous, et non avec nous, un éditeur qui lutte pour sa survie : si Dunod a absorbé Gauthier Villars, il a été lui-même absorbé par Bordas en 1978, d'où l'âpreté des négociations. L'éditeur lèse les intérêts de la SFRP dans deux domaines : la publicité. Le contrat ne se trouve plus dans les archives, mais il semble bien qu'il nous était très défavorable ( $20 \%$ de ristourne sur la publicité, peut être moins). Les abonnements extérieurs : ils ne participent pas à alimenter la société ; là aussi, nous manquons de documentation, mais il semble que les ristournes sur ces abonnements sont faibles, sinon nulles.

S'ajoutent à cela de nombreux griefs : énorme retard dans l'envoi des épreuves aux auteurs pour corrections, incertitude dans le fichier des membres de la société, frais généraux bien souvent injustifiés, et, fait plus grave, utilisation au seul profit de l'éditeur de la subvention du CNRS [8]. 
À la suggestion du conseil d'administration, A. Teste du Bailler recherche, à partir de la fin de 1980, un nouvel éditeur. Il contacte RGE SA, qui édite la revue générale de l'électricité, puis, à la suggestion de Jacques Pradel, le GEDIM (groupe des éditeurs de l'industrie minérale), à Saint-Étienne, qui édite les annales des mines. Si un nouvel éditeur est retenu, le protocole avec Dunod devra être dénoncé avant le $1^{\mathrm{er}}$ juillet 1981. En ce qui concerne la publicité, un contact est pris avec la Régie de publicité industrielle.

En avril 1981, la commission de la revue choisit le GEDIM ; dépendant de l'association des anciens élèves de l'école des mines de Saint-Étienne, le GEDIM est logé dans la maison des mines, sa gestion est celle d'une association régie par la loi de 1901. Le contrat proposé est d'un prix très raisonnable. Reste à résilier le contrat avec Dunod. Le 8 mai 1981, le conseil adopte la motion suivante : «le conseil d'administration félicite $\mathrm{Mr}$ Teste du Bailler pour le dynamisme dont il a su faire preuve. Il confirme le choix de la commission de la revue et lui donne mandat d'entreprendre les négociations pour un projet de convention avec un nouvel éditeur ».

Le contrat avec Dunod prend fin le 31 décembre 1981. Lors du conseil du 19 janvier 1982, le président René Coulon «souligne la mauvaise volonté de l'ancien éditeur Dunod, qui fait traîner la publication du $n^{\circ} 4$ de 1981, et qui laisse entendre, notamment dans ses envois aux libraires, que Radioprotection cesse de paraître [...] Mr Chinardet demande que des démarches soient [...] effectuées auprès des libraires concernés » [9].

Peu de temps après, le GEDIM publie le $\mathrm{n}^{\circ} 1$ de 1982 avec une couverture rénovée, de couleur (vert-jaune), toujours au même format.

Après le départ de Mr Teste du Bailler, le conseil, à l'unanimité, élit Pierre Bovard à la tête de « Radioprotection ». Peu après, P. Bovard recrute un nouveau membre pour la commission de la revue, Jacques Lochard.

Il faut toujours revenir aux textes de base. Voici un passage du rapport moral de Raymond Bardoux, en fin de mandat, devant l'AG de juin 1981. Il résume parfaitement l'époque qui vient d'être étudiée : «(la commission de la revue) a été amenée à prendre un certain nombre d'initiatives, allant dans le sens des objectifs fixés par le conseil d'administration. À partir du sondage lancé l'an passé auprès des lecteurs de la revue, une tendance générale s'est dégagée pour souhaiter que la revue soit à l'avenir plus vivante, plus ouverte aux problèmes d'actualité et plus pratique pour les professionnels de la radioprotection. Par des circulaires largement diffusées auprès des responsables d'entreprises, de laboratoires, de milieux médicaux, d'administrations, par l'envoi d'un numéro-specimen de la revue à un millier de personnes concernées par l'industrie du nucléaire, 
la commission a fait connaître la revue à l'extérieur du cercle habituel des spécialistes, tout en sollicitant des articles et des adhésions. Les premiers échos que l'on peut avoir de ces campagnes s'avèrent dès maintenant encourageants ».

\subsection{Le décès de Norbert Chassende Baroz.}

L'année 1982 est endeuillée par la disparition de notre président fondateur, et membre d'honneur. Il était moins présent dans la commission, mais continuait, malgré des problèmes de santé, à faire un travail remarquable de secrétariat de rédaction. Dans son rapport moral devant l'AG du 2 juin 1969, le président (Blanc, 1969) écrivait : "[...] le général Chassende Baroz se dépense sans compter pour assurer à notre revue la structure administrative et rédactionnelle indispensable. Malgré de sérieux ennuis de santé, il n'a pas ralenti son action, trouvant des solutions à tous les problèmes, corrigeant les épreuves des auteurs, rectifiant certaines erreurs, assurant la liaison avec l'imprimeur et lui rappelant inlassablement - les délais à respecter. Au nom de tous, j’adresse à $\mathrm{Mr}$ Chassende Baroz l'expression de notre chaleureuse reconnaissance et de nos sentiments de profonde et très déférente affection ».

Dans la notice qu'il lui a consacré, H. François (François, 1982) écrit : «au cours de sa retraite, il nous faisait de fréquentes visites et se tenait au courant des nouvelles et des idées en cours. Il était passionné d'archéologie et participait à des fouilles. Il s'intéressait, notamment, à l'évolution des techniques de radio-datation par thermoluminescence, nées de la dosimétrie des rayonnements ».

\section{D'octobre 1982 à septembre 1987 : Pierre Bovard avec Anne-Marie Beau}

P. Bovard prend ses fonctions avec la préparation du n ${ }^{\circ} 4$ de 1982. Sur sa demande, il les quittera cinq années plus tard.

La période est stable, aussi bien en ce qui concerne les rapports avec la hiérarchie de la SFRP qu'en ce qui concerne la commission de la revue, dirigée avec tact. Les peuples heureux n'ont pas d'histoire : l'exposé sera relativement court.

À la fin de 1984, Mme Odile Pennaneac'h (centre de documentation du centre de recherche de Fontenay-aux-Roses) vient seconder A.-M. Beau comme secrétaire de rédaction. J. Lochard devient aussi secrétaire de rédaction. La commission de la revue compte 21 membres. Pourquoi le nombre des secrétaires de rédaction augmente-t-il ? À côté d'une commission qui grossit et devient difficilement mobilisable, se forme un groupe opérationnel de cinq personnes, $\mathrm{P}$. Bovard, A.M. Beau et O. Pennaneac'h, H. Joffre et J. Lochard, constituant une 
sorte de comité de rédaction avant l'heure. Trouver des dates de réunion convenant à 21 membres est difficile, et la commission se réunit moins souvent, venant ainsi se placer en retrait des « opérationnels »; cette tendance s'amplifie avec le temps : la commission devient un collège d'experts, très en amont des tâches quotidiennes.

Dans son rapport moral du 22 juin 1984, le président de la SFRP résume la vie de la revue (Teste du Bailler, 1984): «[...] pour ma part, notre revue Radioprotection semble se plaire dans sa nouvelle présentation. Merci à Mme Beau, toujours fidèle, et j'oserai ajouter "jusqu'à quand? ». Merci à Mr Bovard qui sait dénicher de bons articles. À noter l'initiative d'un numéro spécial sur les RNI (rayonnements non ionisants), qui nous a coûté de l'argent, mais avait pour objectif de nous apporter des sympathies, et peut être des adhésions. À ce propos, je note que le groupe des RNI, avec MM Bertheaud, Le Ruz et bien d'autres, développe de nouvelles ouvertures sur ce problème important ».

Le 11 décembre 1984, la commission de la revue décide de créer officiellement un comité de rédaction. Autour de P. Bovard, trois personnes, Mmes Beau et Pennaneac'h, Mr Lochard, forment le premier noyau. Par la suite, le comité s'agrandira. Le conseil d'administration approuve cette création [10]. Certains souhaitent qu' on en profite pour supprimer les «nouvelles de la radioprotection », bulletin fermement défendu par R. Coulon, F. Duhamel, P. Jockey et A. Teste du Bailler. Le conseil convient que «le comité de rédaction peut aider à la composition du bulletin ».

L'année 1985 est assombrie par le décès de H. François, en juillet, après une courte maladie. La commission de la revue lui consacre un article (collectif, 1985) : "président de la commission des publications jusqu'en 1979, son souci constant fut de hisser la revue à un niveau scientifique qui lui mérite une audience sans cesse accrue auprès de la communauté pluridisciplinaire concernée par la protection contre les rayonnements ionisants et non ionisants [...]. Bourguignon, il en avait toutes les qualités : joie de vivre, ténacité, sens de la terre, du concret».

Le 20 octobre 1986, P. Bovard, retraité depuis quatre ans, fait part de son désir de quitter ses fonctions : il est élu local, et souhaite se consacrer à cette tâche. Sur la proposition de J. Pradel, le conseil élit D. Blanc, qui souhaite que le début de son mandat coïncide avec celui de J. Pradel à la présidence de la SFRP. Le conseil approuve et P. Bovard reste en fonction jusqu'au 23 juin 1987.

\section{De juin 1987 à septembre 1996 : Daniel Blanc avec Anne-Marie Beau (jusqu'à la fin de 1995)}

D. Blanc arrive au moment de la sortie du $\mathrm{n}^{\circ} 3$ de 1987 , le vice président de la commission étant toujours H. Joffre. Il arrêtera en septembre 1996, sa retraite 
universitaire sonnant à la fin de ce mois, mais il demeurera membre du comité de rédaction.

\subsection{L'époque «commission de la revue» jusqu'à la fin de 1992}

D. Blanc débute son mandat par une visite à Saint-Etienne, où il rencontre $\mathrm{Mr}$ Favre Bulle, secrétaire général du GEDIM. Ce dernier propose à la SFRP d'adhérer à ce groupement, qui compte 22 adhérents, dont la COGEMA. En acquittant une cotisation annuelle de $5000 \mathrm{~F}$, la SFRP disposerait de quatre voix sur les 310 de l'AG du GEDIM, et pourrait ainsi s'exprimer. La commission juridique et du règlement fait des réserves; l'avenir montrera qu'elles sont justifiées. Influencé par sa visite, D. Blanc propose de mettre la revue au format A4 $(21 \mathrm{~cm} \times 29,7 \mathrm{~cm})$, présentation courante pour les revues scientifiques, et ce sans incidences financières. Le 2 octobre 1987, le conseil refuse cette proposition, et maintient le format de la revue, qui n'a pas changé depuis.

La commission continue à grossir, en s'enrichissant d'experts dans de nouveaux domaines. En 1992, la commission compte 24 membres français, et 17 membres étrangers. Le comité de rédaction compte six personnes, Mmes A.-M. Beau et O. Pennaneac'h, MM D. Blanc, Christian Lefaure, Jacques Lombard et Henri Métivier.

La commission ne se réunit plus que deux fois par an; chacun donne son opinion, fait des suggestions et des critiques, soigneusement notées. La réunion se termine autour d'un buffet. C'est agréable et utile, mais quelque peu consommateur de temps et d'énergie. Sur la suggestion des «six », après délibération du conseil, le président Laurent Stricker supprime la commission à la fin de 1992, et ne conserve que le comité de rédaction.

Voyons maintenant quels sont les faits marquants de la période 1987-1992.

L'autorisation de publier de articles en anglais - Après avoir restauré l'avance en manuscrits (le stock initial était d'un numéro), on en est revenu à la préoccupation commune à tous les responsables successifs : augmenter l'audience de la revue. D. Blanc demande par deux fois au conseil l'autorisation de publier des articles en anglais ; la discussion est difficile, mais cette autorisation est enfin donnée le 9 octobre 1991 [11], moyennant une proportion de moins de $30 \%$ des pages pour les textes en anglais. Le comité l'interprète comme une proportion annuelle.

Dans son rapport moral pour 1992, le président L. Stricker écrit (Stricker, 1992) : «[...] La revue est, en effet, à la fois un agent de liaison entre nous et un moyen 
de promotion en France et à l'étranger. Le conseil d'administration a décidé d'ouvrir les colonnes de "Radioprotection » à des articles en langue anglaise. Un tel choix n'était pas forcément évident, mais il constitue un tournant important de la revue qui contribuera à donner à la SFRP une audience à caractère international et une ouverture plus large. Cela est possible et également souhaitable. «Radioprotection » participe donc à la promotion de la SFRP ».

Le changement forcé d'éditeur — Le GEDIM annonce, heureusement très en avance, qu'il cessera ses activités le $1^{\mathrm{er}}$ septembre 1991. Cela implique que notre engagement avec le GEDIM s'achèvera fin juin 1991. La raison est l'évolution défavorable des comptes du GEDIM. Nous l'avons déploré, car le travail effectué était excellent, et nous entretenions les meilleurs rapports avec le personnel du GEDIM. Les annales des mines, publication principale du GEDIM, sont reprises par la société ESKA, qui propose à la SFRP un contrat «type GEDIM », mais, alors que nous dépensions 180000 francs par an, le nouvel éditeur, pour les mêmes prestations, propose 266000 francs par an.

Y. Chelet demande un devis aux Éditions de Physique, avenue du Hoggar, Les Ulis : le prix est de 190000 francs. Le conseil examine la question le 6 mars 1991 [12]. Comme on pouvait s'y attendre, les Éditions de Physique ont la préférence. Cette société, émanation de la Société française de physique, mais financièrement indépendante, est spécialisée dans la réalisations de revues scientifiques et techniques de haut niveau (dont les diverses éditions du journal de physique); nous y avons trouvé une solide compétence, et des prix raisonnables, ne mettant pas en péril notre budget. Le publiciste reste le même (la régie de publicité industrielle).

La promotion de la revue - En 1991, nous fêtons le $25^{\mathrm{e}}$ anniversaire de la revue en publiant un numéro plus abondant. Préfigurant l'avenir, sous l'impulsion de H. Métivier fut joué un coup heureux, avec l'appui efficace de Mme Berger, directrice des éditions de physique : bien que l'organisateur ait pratiquement sélectionné un éditeur allemand, nous sommes parvenus, au dernier moment, grâce à des prix attractifs, à le faire revenir en arrière, et à obtenir le contrat de réalisation des actes du congrès de Fribourg (15-18 septembre1992), «impact des installations nucléaires sur l'environnement ». La couverture reproduisait celle de la revue, et ce fut pour nous une belle opération publicitaire.

En 1992, le « look » de la couverture est modifié. Un fond rouge, entouré d'un liseré vert, évoque le rôle de protection de notre discipline. L'accueil des lecteurs fut d'abord mitigé : toute nouveauté choque. Une nouvelle modification, en 1993, portant sur le sigle, fut appréciée. L'unanimité fut acquise avec la présentation du $\mathrm{n}^{\circ} 1$ de 1995. C'est la couverture actuelle. 


\subsection{Depuis 1993 : l'époque «comité de rédaction"}

Le comité de rédaction est maintenant en liaison directe avec le conseil et le président. Rappelons qu'il compte six membres, Mmes A.M. Beau, O. Pennaneac'h, MM D. Blanc, C. Lefaure, J. Lombard et H. Métivier. Le nombre des experts du comité est trop faible pour qu'ils puissent englober tous les domaines; la désignation des rapporteurs et des lecteurs extérieurs les plus compétents est une tâche difficile pour eux. Pour cette raison, sont cooptés dans le comité Henri Magdelenat (institut Curie) et Georges Tymen (université de Bretagne occidentale, Brest). Par ailleurs, H. Métivier devient vice président du comité. J. Lombard devient secrétaire général de la SFRP lors de l'AG de juin 1995 ; il garde sa place dans le comité, mais « es qualité ».

Des numéros supplémentaires — Plusieurs numéros supplémentaires marquent cette période. Le plus marquant est «épidémiologie et rayonnements ionisants »; ses 121 pages ont coûté beaucoup de travail et de ténacité ; il est distribué avec le $\mathrm{n}^{\circ} 3$ de 1994. Il est annoncé par le président Jean-Marc Cosset, lors de l'AG du 20 septembre 1994 (Cosset, 1994) : "la commission de la revue (lire : le comité de rédaction) s'améliore, notamment par sa qualité et la diversité des articles publiés. Les lecteurs ont manifestement accueilli de manière favorable la rubrique "lettre de l'Europe ». Les temps forts prévus pour Radioprotection vont être la sortie d'un certain nombre de numéros spéciaux. Le premier, qui concerne l'épidémiologie, est attendu, et devrait être de bonne qualité ».

En effet, la « lettre de l'Europe », animée par H. Métivier, a vu le jour avec le $\mathrm{n}^{\circ} 4$ de 1993. H. Métivier conduisait la délégation française auprès du comité de gestion et de coordination de la DG 11 (CGC 10); elle reçut un excellent accueil. Hélas, la suppression ultérieure du CGC 10 a conduit à l'arrêt de cette chronique, avec le $\mathrm{n}^{\circ} 1$ de 1995.

La naissance de « RP magazine » — Les « nouvelles de la radioprotection » ont disparu depuis longtemps ; leur contenu a été rapatrié dans la revue, mais sans frontière définie avec la partie purement scientifique. Lors du conseil du 14 décembre 1994 [13], « Mr Lochard souhaite que les articles soient une source d'information sur la radioprotection. Par ailleurs, il préconise de changer la présentation de la revue. Celle-ci doit comporter deux parties, la première correspondant à un magazine d'information, la seconde contenant les articles bien séparés ».

Sur la proposition de J. Lombard, le 3 novembre 1995 [14], le conseil décide que : "la revue est divisée en deux parties, l'une scientifique, l'autre de type magazine. Cette dernière partie sera préparée par le secrétaire général ». 
Les éditoriaux - A. Teste du Bailler avait lancé la mode éditoriale ; ce fut irréversible. D. Blanc en écrit 21... Sur l'impulsion de H. Métivier, le comité publie, en éditorial du n ${ }^{\circ} 2$ de 1995, un bilan de santé portant sur cinq années, 1990 à 1995 incluse ; on est loin des angoisses ressenties une vingtaine d'années auparavant.

Dans son dernier éditorial (Blanc, 1996), D. Blanc rappelle l'incendie du vendredi 28 juin 1996, qui a gravement endommagé le bureau de H. Métivier. Il put cependant sauver les documents concernant la revue, dont la publication ne fut pas retardée. Cet éditorial explique le trajet d'un manuscrit, de son envoi par l'auteur à sa publication dans la revue : "Voyons comment vit un article envoyé au secrétariat de la SFRP : les articles arrivent au secrétariat, puis reçoivent, chez Henri Métivier, un numéro d'enregistrement. Un accusé de réception est envoyé aux auteurs. Dans le même temps, notre vice-président suggère à tous ses collègues du comité de rédaction un nom de rapporteur et trois noms de lecteurs; il envoie à chaque membre du comité une copie de l'article.

Si les membres sont d'accord, le texte est envoyé, 8 à 10 jours après réception, aux lecteurs, qui ont un mois pour donner leur avis. Ces avis sont regroupés et synthétisés par le rapporteur, qui écrit un rapport circonstancié et l'adresse au comité de la revue. Viennent ensuite les éventuelles corrections - assez. fréquentes - Si les corrections à effectuer restent importantes, l'article corrigé est de nouveau soumis au rapporteur. Ce n'est qu'à ce moment que l'article sera, ou ne sera pas, accepté.

Depuis le départ de Mme Beau (voir plus loin), une chose a changé ; les articles doivent être envoyés aux Éditions de physique sous forme de disquette. [...] Ce n'est qu'à ce prix que nous avons pu négocier une baisse significative des coûts de fabrication avec les éditions de physique, dans une période où les coûts de production de l'écrit augmentent. Cette maîtrise des coûts est une condition de survie pour notre revue, qui, nous l'espérons, continuera à vous satisfaire ».

Départs - Madame Beau avait pris sa retraite en 1993, mais elle continuait son travail de secrétaire de rédaction. Elle décide de prendre une complète retraite, et nous quitte à la fin de 1995. Dans l'éditorial du nº 1 de 1995 D. Blanc écrit : [...] Son activité dans le cadre de notre revue a été d'une exceptionnelle qualité, car rien ne lui échappait dans l'élaboration des numéros successifs. Elle assurait également la rédaction de la partie "informations » de la revue, avec les annonces des cours et des congrès, et sans rien omettre de tous les détails souhaitables. Cette partie de la revue va être prise en charge par le secrétariat général. Mme Beau réalisait un travail remarquable de corrections d'épreuves. Peut être ne l'avez-vous pas remarqué : notre revue comporte un nombre extrêmement faible de fautes d'impression. Par ailleurs, les instructions données aux auteurs sont 
appliquées de manière très rigoureuse. Ces qualités éditoriales, nous les devons à Mme Beau. Nous allons nous efforcer de continuer à bien faire [...] ».

Au conseil du 3 novembre 1995 [14], Mme Annie Sugier fait part du désir de D. Blanc de mettre fin à ses fonctions à la fin de septembre 1996; il propose d'être remplacé par H. Métivier. Le conseil approuve et suggère que MM Blanc et Métivier assistent simultanément aux réunions du conseil pendant la période transitoire. Le 3 octobre 1996 [15], "sur proposition de D. Blanc, président en exercice du comité la revue [...], Henri Métivier est nommé président du comité de la revue à compter de ce jour et pour une durée de trois ans (octobre 1996-octobre 1999)».

\section{Depuis octobre 1996 : Henri Métivier et collaborateurs}

Nous limiterons notre propos à la fin de 1999. Ensuite, c'est la période contemporaine.

Les problèmes posés par le départ de Mme Beau ont été résolus par la division du travail. Le secrétariat de rédaction demeure près d'H. Métivier, il assure aussi la liaison avec les Éditions de Physique, représentées dans le comité de rédaction par Florence Grosseaux (jusqu'en juillet 1998), puis par Agnès Henri. La directrice de la publication, Mme Berger, prend sa retraite à la fin de 1996 ; elle est remplacée par Jean-Marc Quilbé. La partie «RP magazine » est rédigée par un certain nombre de chroniqueurs, dont $\mathrm{P}$. Ballereau pour la réglementation. La coordination est assurée par J. Lombard et ses deux collaboratrices, Mmes Patricia Piqueras et Janine Cervera.

Le succès de cette nouvelle présentation est confirmé dans le rapport moral de la présidente A. Sugier devant l'AG du 18 juin 1997 à Avignon (Sugier, 1997) : [...] Il fallait aussi que la revue de la SFRP soit non seulement le véhicule d'articles scientifiques mais aussi le support de la vie de la société avec une partie magazine renforcée notamment par des textes d'actualité et des interviews. Je ne suis pas peu fière d'avoir bénéficié des talents d'un jeune retraité et nouveau chroniqueur, Jérôme Pellissier-Tanon ».

Enfin, la SFRP crée son site à compter du $1^{\mathrm{er}}$ janvier 1998, www.sfrp.asso.fr.

L'harmonie qui règne entre le comité de rédaction et le conseil de la SFRP est évoquée par le président Jacques Lochard, sans son rapport moral du 9 juin 1998, à La Rochelle (Lochard, 1998) : "Je voudrais signaler la qualité du travail de la commission de la revue Radioprotection. Grâce à elle, la revue a bien évolué ces deux dernières années, et est parvenue à un bon équilibre entre les aspects 
technico-scientifiques et la partie magazine. Cette dernière contient des informations sur la vie de l'association et les entretiens très appréciés de $\mathrm{Mr}$ Jérôme Pellissier-Tanon. J'encourage tous les membres de la SFRP à contribuer à la revue Radioprotection ».

\section{Note importante}

Aux références, présentées selon les instructions aux auteurs, s'ajoute une liste de documents non publiés, cités dans le texte : [1] document 71/2280, PV du CA du 2 avril 1971, [2] document 71/2316, PV du CA du 2 juin 1971, [3] document 72/2483,PV du CA du 27 janvier 1972, [4] document 72/2569, PV du CA du $1^{\text {er }}$ juin 1972, [5] document 74/228, PV du CA du 28 novembre 1973, [6] document 78/123, PV du CA du 10 novembre 1978, [7] document 79/086, PV du CA du 24 septembre 1979, 4-5, [8] document 81/003, PV du CA du 5 janvier 1981, [9] document 80/82, PV du CA du 19 janvier 1982, [10] document 91/84, PV du CA du 12 décembre 1984, [11] document 120 90/91, PV du CA du 9 octobre 1991, [12] document 117 90/91, PV du CA du 6 mars 1991, [13] PV du CA du 14 décembre 1994, [14] PV du CA du 3 novembre 1995, [15] PV du CA du 3 octobre 1996.

\section{RÉFÉRENCES}

Blanc D. (1969) rapport moral, Radioprotection 14, 251.

Blanc D. (1996) éditorial, Radioprotection 31, 335-336.

Collectif (1985) Radioprotection 20, 281.

Cosset J.M. (1994) rapport moral, Radioprotection 29, 613.

Delpla M. (1968) rapport moral, Radioprotection 3, 160-162.

Duhamel F. (1970) rapport moral, Radioprotection 5, 251-255

Fitoussi L. (1977) rapport moral, Radioprotection 12, 290-295.

François H. (1982) Radioprotection 17, 213-214.

Gey A. (1974) rapport moral, Radioprotection 9, 154-158.

Letard H. (1976) rapport moral, Radioprotection 11, 237-240.

Lochard J. (1998) rapport moral, Radioprotection 33, 346.

Stricker L. (1992) rapport moral, Radioprotection 27, 352.

Sugier A. (1997) rapport moral, Radioprotection 32, 403.

Teste du Bailler A. (1984) rapport moral, Radioprotection 19, 253-255. 